\title{
Hair-Structure Mystery Solved by Datamining Two Decades of Electron Tomograms
}

Duane P. Harland $^{1 *}$, Veronika Novotná ${ }^{1,2}$, Marina Richena ${ }^{1}$, Mihnea Bostina ${ }^{3}$, Sailakshmi Velamoor ${ }^{1,3}$ and A. John McKinnon ${ }^{4}$

${ }^{1 .}$ Food and Bio-based Products Group, AgResearch, Crown Research Institute, Lincoln, New Zealand.

2. Power Electrical and Electronic Engineering, Brno University of Technology, Brno, Czech Republic.

3. Microbiology and Immunology, University of Otago, Dunedin, New Zealand.

4. Institute of Fundamental Sciences, Massey University, Palmerston North, New Zealand.

* Corresponding author: duane.harland@agresearch.co.nz

The main structural component of hair shafts is the cortex. Like all components of hair, the cortex is composed of sacrificed cell remnants filled with cornified keratin. In cortical cells, the main keratin building block is the macrofibril. These are long bundles of keratin intermediate filaments (KIFs), which are about $500 \mathrm{~nm}$ in diameter and of uncertain length. The KIFs can be helically arranged around the macrofibril axis, with KIF helical pitch increasing linearly from macrofibril core to edge [1].

Hair growth, and thus macrofibril formation occurs within follicles in the skin. Early stages of macrofibril formation appears to be largely a mesophase-based self-assembly process in which the precursors of macrofibrils first appear as liquid-crystal tactoids, and this is driven initially by interactions between pre-keratinized $10 \mathrm{~nm}$ diameter KIFs [2, 3]. Differences in the nature of these KIFKIF interactions during self-assembly can result in different proportions of macrofibrils with different twist handedness. Our objective was to establish if macrofibrils are all a single handedness or if they form 50:50 mixtures of handedness within each cortical cell. Earlier work from our laboratory, in which we modelled the structure of single macrofibrils using electron tomography data suggested that both left and right-handed forms exist.

We data-mined 41 electron tomograms containing three-dimensional macrofibril data from previously published studies of Japanese [4] and Caucasian [5] scalp hair, and also different types of wool [6-8]. The data was from MRC files that had been processed using eTomo and the IMOD suite of tools (https://bio3d.colorado.edu/imod/). The tomograms were from tilt series collected on several different $300 \mathrm{kV}$ TEMs from 1999 to 2011. In all, using IMOD (v4.5.9) we examined 644 macrofibrils and found that within any specific tomogram, that all macrofibrils had the same handedness. Due to the pattern of results we concluded that earlier reports of left and right handed macrofibrils were due to artefacts of imaging or data processing.

To validate our initial conclusion, we used a handedness marker composed of DNA origami decorated with a left handed helix of gold nanoparticles (GATTAquant GmbH, Braunschweig, Germany) [9] and also re-imaged some of the original sections from earlier studies (JEM-2200FS Cryo-TEM, JEOL, Japan, with a TVIPS-TemCam F416, TVIPS-GmbH, Germany; 200 kV, ×25,000 Magnification; Serial EM 3.4 and eTomo 4.9.9) to establish that in all cases all macrofibrils are left-handed around the macrofibril axis (Figure 1). With agreement within human samples and sheep, we provisionally conclude that this state is universal within mammalian hair. This also supports the conclusion that the origin of macrofibril twist is the expression of chiral twisting forces between adjacent KIFs, rather than 
mesophase splay and bending forces relaxing to twisting forces acting within a confined space (such as a cortex cell).

Importantly the current work shows:

1) the immense value of archiving tomography data;

2) the importance of standardised file types for data, in this case the MRC format;

3) the value of archived sectioned material.

Furthermore, the question of handedness can be an issue in transmission electron microscopy. Our results suggest that keratin samples may, in some circumstances, be used to test if images have been mirror-reflected.

References:

[1] JE Plowman and DP Harland in "The Hair Fibre: Proteins, Structure and Development”, ed. JE Plowman, DP Harland and S Deb Choudhury, (Springer, New York) p. 3.

[2] DP Harland and AJ McKinnon in "The Hair Fibre: Proteins, Structure and Development", ed. JE Plowman, DP Harland and S Deb-Choudhury, (Springer Nature, Singapore) p. 155.

[3] J McKinnon and DP Harland, Journal of Structural Biology 173 (2011), p. 229.

[4] WG Bryson et al., Journal of Structural Biology 166 (2009), p. 46.

[5] DP Harland et al., Journal of Structural Biology 185 (2014), p. 397.

[6] WG Bryson et al., (2000) p. 1.

[7] JP Caldwell et al., Journal of Structural Biology 151 (2005), p. 298.

[8] DP Harland et al., Journal of Structural Biology 173 (2011), p. 29.

[9] A Briegel et al., Journal of Structural Biology 183 (2013), p. 95.
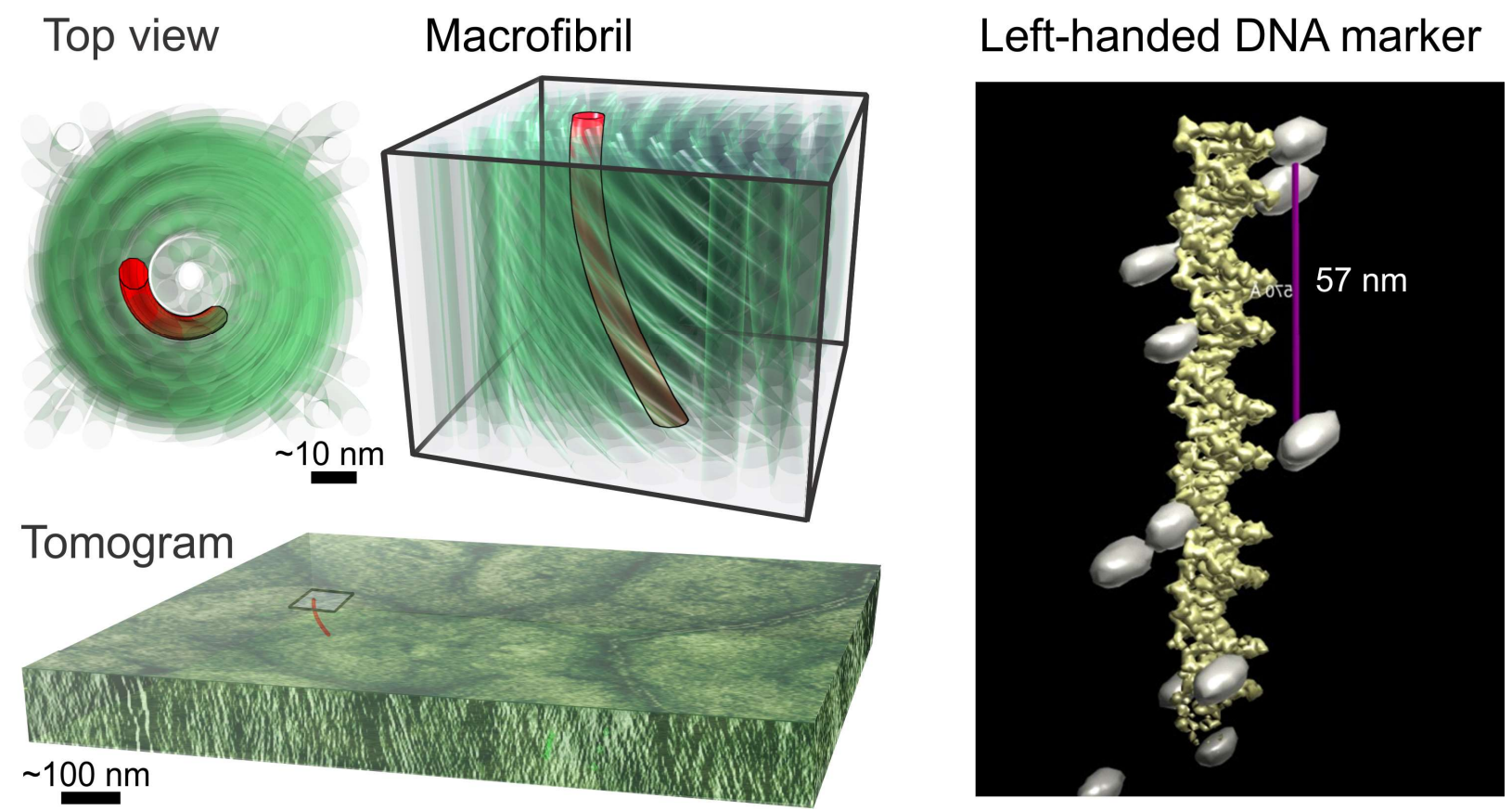

Figure 1. Left, Illustrative model of a left-handed macrofibril (top and perspective), a tomogram, highlighting a single keratin intermediate filament. Right, handedness-marker, showing locations of tomographically imaged gold markers fitted to a virtual DNA molecule. 